\title{
Understanding variation in length of hospital stay for COPD exacerbation: European COPD audit
}

\author{
Mamta Ruparel ${ }^{1}$, Jose Luis López-Campos ${ }^{2,3}$, Ady Castro-Acosta ${ }^{3,4}$, \\ Sylvia Hartl ${ }^{5}$, Francisco Pozo-Rodriguez ${ }^{3,4}$ and C. Michael Roberts ${ }^{6}$ on behalf \\ of the European COPD Audit ${ }^{7}$
}

\begin{abstract}
Affiliations: 'University College London, London, UK. ${ }^{2}$ Unidad Medico-Quirúrgica de Enfermedades Respiratorias/Instituto de Biomedicina de Sevilla (IBiS), Hospital Universitario Virgen del Rocío/Universidad de Sevilla, Seville, Spain. ${ }^{3}$ Centre for Biomedical Research on Respiratory Diseases (CIBERES), Instituto de Salud Carlos III, Madrid, Spain. ${ }^{4}$ Instituto de Investigación, Hospital 12 de Octubre, Madrid, Spain. ${ }^{5}$ Ludwig Boltzmann Institute of COPD and Respiratory Epidemiology, Otto Wagner Hospital, Vienna, Austria. ${ }^{6}$ Barts and The London School of Medicine and Dentistry, Queen Mary, University of London, London, UK. ${ }^{7}$ For a list of the European COPD Audit team members and their affiliations, see the supplementary material.
\end{abstract}

Correspondence: Mamta Ruparel, University College London, Division of Medicine, 5 University Street, London WC1E 6JF, UK. E-mail: m.ruparelducl.ac.uk

ABSTRACT Chronic obstructive pulmonary disease (COPD) care across Europe has high heterogeneity with respect to cost and the services available. Variations in length of stay (LOS) may be attributed to patient characteristics, resource and organisational characteristics, and/or the so-called hospital cluster effect.

The European COPD Audit in 13 countries included data from 16018 hospitalised patients. The recorded variables included information on patient and disease characteristics, and resources available. Variables associated with LOS were evaluated by a multivariate, multilevel analysis.

Mean \pm SD LOS was $8.7 \pm 8.3$ days (median 7 days, interquartile range $4-11$ days). Crude variability between countries was reduced after accounting for clinical factors and the clustering effect. The main factors associated with LOS being longer than the median were related to disease or exacerbation severity, including GOLD class IV (OR 1.77) and use of mechanical ventilation (OR 2.15). Few individual resource variables were associated with LOS after accounting for the hospital cluster effect.

This study emphasises the importance of the patients' clinical severity at presentation in predicting LOS. Identifying patients at risk of a long hospital stay at admission and providing targeted interventions offers the potential to reduce LOS for these individuals. The complex interactions between factors and systems were more important that any single resource or organisational factor in determining differences in LOS between hospitals or countries.

@ERSpublications

LOS determined by COPD exacerbation severity and interactions within hospitals constituting effective patient care http://ow.ly/XD9hy

This article has supplementary material available from openres.ersjournals.com

Received: Jun 152015 | Accepted after revision: Jan 182016

Conflict of interest: Disclosures can be found alongside this article at openres.ersjournals.com

Copyright $\odot$ ERS 2016. This article is open access and distributed under the terms of the Creative Commons Attribution Non-Commercial Licence 4.0. 


\section{Introduction}

Chronic obstructive pulmonary disease (COPD) is a condition with high morbidity and cost to health services. Hospitalisations due to COPD negatively affect the well-being of patients with an impact on length and quality of life. Studies in different European countries have highlighted the cost of the disease and that of admissions. In a survey conducted in 1998-1999, annual direct expenditure for COPD treatment per patient in Europe ranged from $€ 530$ in France to $€ 3238$ in Spain [1] with a significant proportion of this cost due to inpatient treatment of exacerbations [1,2]. Accordingly, several initiatives have been proposed to reduce the number of hospitalisations or to reduce the length of stay (LOS) from a cost-effectiveness and clinical perspective [3-5].

Various factors have been implicated in increasing LOS of hospital admissions, both clinical [6-10] and social [11]. European health systems have a large inter-hospital variability in terms of resources and the range of services offered [12,13], and some previous reports have found a relationship between LOS and such factors $[4,14,15]$, whilst others have not $[16,17]$. To our knowledge, there has been no comprehensive analysis studying the effects of clinical, organisational and resource factors on LOS in European patients admitted with exacerbations of COPD across a range of European countries and healthcare systems.

The European COPD Audit was a European Respiratory Society (ERS)-funded project designed as a pilot study, performed in 2010-2011 to evaluate clinical practice variability as well as clinical and organisational factors related to outcomes for COPD hospital admissions across Europe, in which 432 hospitals from 13 European countries participated including 16018 cases of COPD exacerbations admitted to hospital.

In the present analysis, we aimed to determine the mean LOS across Europe and determine the degree of variability to test the hypothesis that these variations in LOS may be attributed to either patient characteristics or the organisational and resource characteristics, and determine the relative importance of these factors. We also hypothesised that LOS may be influenced by the hospital cluster effect, which indicates that patients with similar characteristics may experience different processes of care and outcomes, depending on the hospital to which they are admitted. These include a wide range of complex factors not easily measured that might influence clinical care including local guidelines and policies, practices of individual clinicians, the admission policy, cultural practices that may influence the decision whether to admit or discharge patients, or clinical characteristics that may be observed within geographical clusters. These include factors common to the local population, such as socio-demographics, family and social support networks, cultural beliefs and attitudes, and environmental susceptibilities. Such effects on patient outcomes, although not measured individually, can be combined in a theoretical statistical model as the cluster effect to explain residual variation once the factors that have been measured have been accounted for.

\section{Methods}

The study design and the methodology of the European COPD Audit have been extensively described elsewhere [18]. Briefly, this ERS-funded COPD audit was designed as a pilot study to evaluate clinical practice variability as well as clinical, organisational and resource factors related to outcomes for COPD hospital admissions in 13 countries across Europe (Austria, Belgium, Croatia, Greece, Ireland, Malta, Poland, Romania, Slovakia, Spain, Switzerland, Turkey and the UK). It was designed as a prospective, observational, non-interventional cohort trial.

The study comprised of a first 8-week phase during which all consecutive cases admitted to hospital for greater than $12 \mathrm{~h}$ due to an exacerbation of COPD were identified. These were considered potential cases, which were then confirmed as an exacerbation upon discharge, according to the diagnosis on the discharge report. Information on clinical practice was gathered for these patients. Comorbidities were recorded as a Charlson Comorbidity Index score, a validated measure of comorbidity status [19]. During this period, participating hospitals were also asked to complete a cross-sectional survey of the resources devoted to COPD acute care. Cases included had their medical history regarding the admission audited, and they were followed-up for 90 days after the admission to evaluate readmissions and survival.

The ERS managed the audit and named a steering committee, which reported to the ERS Executive Committee, to oversee the process. Items for the organisational and resource database were selected by the steering committee, based upon those that had previously been used and validated in the Spanish and UK national audits $[20,21]$. The audit included questions related to the hospital and to the respiratory unit resources separately. An item list of the final survey is available [18].

The study period comprised hospital admissions from October 25, 2010, to December 19, 2010, and from January 3, 2011, until February 27, 2011, according to the seasonal COPD exacerbation peak of the participating countries. As there is no European ethics committee for audits; national societies ensured compliance with European and national ethical requirements, and all participants followed the European ethical requirements for scientific studies. All partners of the project accepted the general ethical rules of the ERS. 
A software company (IDCode, Lausanne, Switzerland) was commissioned to design a web-based collection tool encompassing both the resource and organisational as well as clinical databases. The anonymised data was entered remotely at each participating site to a centrally controlled server. The web tool was established as a multilingual database, to allow the data to be documented in the language of each participating country. The web tool provided a "help facility" with explanatory text to assist with interpretation of the survey questions.

\section{Data analysis}

Statistical analysis was performed by a data-analysis team in Spain using Stata 12 (StataCorp LP, College Station, TX, USA). Descriptive variables were summarised by using the mean \pm sD for quantitative variables and the absolute (relative) frequencies for categorical ones.

Since LOS did not follow a normal distribution, the variables were characterised and those patients with a LOS beyond the median were further studied. Associations of variables potentially associated with LOS were tested by using a multivariable, multilevel logistic regression analysis for LOS longer than the median as the dependent variable. Continuous variables were categorised in different strata in which a missing value was considered one independent category. As part of an audit, missing information is also relevant. By categorising missing values, it allowed us to control the effect of one missing piece of information in the clinical performance. The only exceptions were age and Charlson index, which were not categorised since there was no missing information in these variables. In this multilevel model, data are analysed considering the hierarchical distribution of data, taking into consideration if the variables represent hospital characteristics (level 1) or patient characteristics (level 2). These analyses were performed with the following assumptions: 1) the multilevel model assumes a hierarchical data structure with explanatory variables measured at both hospital and patient levels; and 2) inter-hospital variation in the dependent variable was random.

In this analysis, we acknowledged that patients within the same hospital may receive similar care, which might be different from the care they may receive in other hospitals for the same clinical situation. This is so because patients within one hospital are subjected to distinct common contextual, hospital-linked influences. Our hypothesis is that this different behaviour in the different participant hospitals, the so-called hospital cluster effect, may influence LOS. Multivariable, multilevel regression analysis enables quantification of this hospital cluster effect and provides unbiased estimates of the regression coefficients. It can also concurrently include the predictor variables at both the patient level and hospital level. The coefficients of the predictor variables were transformed into odds ratios with $95 \%$ confidence intervals.

In addition, the hospital cluster effect was evaluated and quantified by two indicators: 1) the intra-cluster correlation coefficient (ICC) adapted to the logistic regression [22] and 2) the median odds ratio (MOR) with $95 \%$ confidence intervals $[23,24]$. The ICC quantifies the fraction of the total variability in the outcomes attributable to the clustering effect. The ICC ranges from 0 to 1 , and a lower ICC indicates a smaller cluster effect. The MOR is a measure of cluster heterogeneity and is defined as the median value of the distribution of odds ratios obtained when two individuals from different hospitals (i.e. one individual from the highest LOS hospital and one individual from the lowest LOS hospital) were randomly selected and compared. Simplified, the MOR can be interpreted as the median increased odds of reaching the outcome if an individual was admitted to another hospital with a greater risk of that outcome. The measure is always $\geqslant 1$, where the MOR equals 1 when there is no between-cluster variation and the MOR increases as the variation becomes larger. Interestingly, the MOR is statistically independent of the prevalence of the outcome, can be easily computed, and is directly comparable with the coefficients of the individual- and hospital-level predictors. To plot the reduction in the between-hospital outcome variations after accounting for the hospital cluster effect and the predictor variables, the crude estimates of probability were transformed to the logit scale for comparisons, and cases with no LOS recorded were excluded.

For the multilevel analysis, we first fitted an "empty model" that only considered the clustering effect on the variability of LOS, expressed as ICC and MOR. Backward selection was attempted; however, the data did not converge in the multilevel analysis because of the high number of variables included so we opted for the forward selection procedure, which provided a better fit. The "final model", therefore, used a forward selection procedure based on Wald tests results, added as covariates to the model those "explanatory variables" associated with the outcome of interest in the bivariate analysis.

Various approaches to handle missing data were considered including analysis of the data without considering the missing information. This was rejected as in the multivariable analysis, a large number of cases would have been deleted due to missing values for different variables, which would have led to a shorter and biased database. Imputation was also considered; however, again, it was felt that this would result in a dataset that would again be artificial and not representative of our sample. Therefore, we considered the "missing values" as an additional category of those variables. 


\section{Results}

During the study, 19150 cases were initially considered upon admission as potential cases. Of these, the diagnosis of COPD exacerbation was confirmed by the lead investigator in that centre in 16018 cases upon discharge. However, in 37 cases, the LOS was not possible to calculate due to lack of information. Thus, the sample for the present analysis was 15981 patients. Mean \pm SD LOS was $8.7 \pm 8.3$ days (median 7 days, interquartile range 4-11 days). The crude distribution of the LOS between countries is presented in table 1 in the supplementary material. Of the analysed population, 6955 (43.4\%) cases had a LOS longer than the median. The description of the patient- and disease-related variables are summarised in table 1 . The sample of patients had high pack-year smoking histories (mean 47.2 pack-years). Forced expiratory volume in $1 \mathrm{~s}$ was skewed towards more severe disease (mean $44 \%$ predicted) and a large proportion had features of infection (50\% had sputum colour change) or co-existent lung consolidation (18.5\%). The organisational variables within the sample varied significantly. Only $31.3 \%$ of units had an early supported discharge service and $48.8 \%$ had access to a pulmonary rehabilitation service. The hospital size and the numbers of respiratory specialist consultants, nurses, lung function technicians and physiotherapists varied greatly.

The bivariate analysis is summarised in the supplementary material (tables $1 \mathrm{~S}$ and $2 \mathrm{~S}$ ). Resource and organisational variables associated with an increased LOS beyond the median were size-related (hospital size by number of beds or the number of pulmonologists), staff-related (having a respiratory team or the number of lung function technicians) and performance-related (the percentage of patients seen by a physiotherapist or the percentage of eligible patients included in a rehabilitation programme). Patient-related variables associated with an increased LOS beyond the median were related to comorbidities, the severity of the exacerbations and interventions given.

\section{TABLE 1 Characteristics of the studied patients}

\begin{tabular}{|c|c|c|}
\hline Variable & Value & Intercountry range \\
\hline Male sex & $10865(67.8)$ & $47.3-86.0$ \\
\hline Age years & $70.7 \pm 10.7$ & $63.4-72.7$ \\
\hline Current smokers & $5012(31.3)$ & $15.4-46.8$ \\
\hline Tobacco history pack-years & $47.2 \pm 31.2$ & $29.8-70.8$ \\
\hline \multicolumn{3}{|l|}{ Comorbidity } \\
\hline Charlson Comorbidity Index score & $2.4 \pm 1.6$ & 1.7-3.1 \\
\hline Cardiovascular disease & $6491(40.5)$ & $32.4-71.4$ \\
\hline Diabetes & $3181(19.9)$ & $11.9-27.5$ \\
\hline Neoplasms & $1950(12.2)$ & $2.6-18.9$ \\
\hline Body mass index $\mathrm{kg} \cdot \mathrm{m}^{-2}$ & $26.6 \pm 6.4$ & $25.0-28.5$ \\
\hline Admissions in previous 12 months & $1.2(1.8)$ & $0.4-1.8$ \\
\hline \multicolumn{3}{|l|}{ Spirometry } \\
\hline FVC $\%$ predicted & $65.4 \pm 20.3$ & $45.2-74.9$ \\
\hline FEV $1 \%$ predicted & $44.0 \pm 17.4$ & $38.9-56.1$ \\
\hline \multicolumn{3}{|c|}{ Previous GOLD spirometric classification } \\
\hline No spirometry available & $6512 \pm 40.7$ & $9.9-53.6$ \\
\hline Spirometry with no obstruction & $1226 \pm 7.7$ & $3.2-34.0$ \\
\hline Mild & $195 \pm 1.2$ & $0-3.0$ \\
\hline Moderate & $2175 \pm 13.6$ & $2.7-31.0$ \\
\hline Severe & $3737 \pm 23.3$ & $11.6-34.9$ \\
\hline Very severe & $2098 \pm 13.1$ & $4.8-23.8$ \\
\hline Missing & $75 \pm 0.5$ & $0-2.7$ \\
\hline Dyspnoea increase & $15409(96.2)$ & $92.5-100$ \\
\hline Sputum increase & $10373(64.8)$ & $49.2-79.0$ \\
\hline Sputum colour change & 8134 (50.8) & $36.9-63.3$ \\
\hline pH & $7.39 \pm 0.07$ & $7.37-7.41$ \\
\hline $\mathrm{PaCO}_{2} \mathrm{kPa}$ & $6.4 \pm 2.1$ & $5.7-7.0$ \\
\hline $\mathrm{PaO}_{2} \mathrm{kPa}$ & $8.6 \pm 3.4$ & $7.3-10.8$ \\
\hline \multicolumn{3}{|l|}{ Chest radiography } \\
\hline Normal or COPD-like findings & 8051 (50.3) & $33.0-55.5$ \\
\hline Consolidation & $2969(18.5)$ & $8.9-32.6$ \\
\hline Other findings & $4752(29.7)$ & $16.7-58.0$ \\
\hline
\end{tabular}

Data are presented as $\mathrm{n}(\%)$ or mean \pm SD, unless otherwise stated. FVC: forced vital capacity; FEV1: forced expiratory volume in $1 \mathrm{~s}$; GOLD: Global Initiative for Chronic Obstructive Lung Disease; $\mathrm{PaCO}_{2}$ : arterial carbon dioxide tension; $\mathrm{PaO}_{2}$ : arterial oxygen tension; $\mathrm{COPD}$ : chronic obstructive pulmonary disease. 
The empty model exhibited a significant hospital cluster effect (ICC 0.18) and cluster heterogeneity (MOR 2.30). In essence, $18 \%$ of the variability was explained by clustering resulting in a 2.3 -fold increased association with LOS. The adjusted model retained a number of variables as outcome predictors, and these were split into the five categories: 1) patient-related variables, 2) disease-related variables, 3) treatment-related variables, 4) exacerbation severity-related variables, and 5) resource-related variables; and the odds ratios depicting the likelihood of the association are in table 2. The strongest relationships were seen between advanced Global Initiative for Chronic Obstructive Lung Disease (GOLD) status (OR 1.77 for GOLD class IV) and the use of mechanical ventilation (OR 2.15). The use of antibiotics and the presence of consolidation on the chest radiography were also strongly associated with LOS (OR 1.46 and 1.27, respectively). Although there was a crude variability of LOS between the different countries, this was reduced after accounting for these clinical factors and the clustering effect (figure 1). The graphical representation of significant variables is depicted in figure 2 .

\section{Discussion}

This study is a comprehensive analysis of factors associated with LOS for COPD patients admitted to hospital with an exacerbation across Europe. Other clinically relevant outcomes including death and readmissions have been previously published elsewhere [25]. The mean and median LOS across Europe were higher than expected from previous studies in individual countries around Europe [10, 26] and showed great variability. This is in keeping with a recent systematic review that showed consistently high variability of COPD LOS across countries in Europe, North America and Australasia [27]. The variability observed in the present study was, however, reduced when the hospital cluster effect (an important consideration in reducing bias from intrinsic institutional factors not measured in the analysis [24]) was accounted for. Clinical and patient factors were associated with LOS but resource and organisational factors had less influence individually than expected from previous studies.

\section{Patient-related factors}

In contrast to previous studies $[9,28]$, the variables of an increase in Charlson index by 1 point, increasing age and body mass index (BMI) classified as overweight or obese were not strongly associated with longer LOS. However, there was a suggestion within the trend that those who had a low BMI (OR 1.13, 95\% CI 0.97-1.33) were more likely to have a longer LOS. This is in accordance with previous studies demonstrating that nutritional status is of importance in quality of life, respiratory muscle strength and exercise capacity $[29,30]$, all factors which would affect recovery from an exacerbation. More recently, an American study reported a reduction in LOS, readmission rate and hospitalisation cost associated with oral nutritional supplementation and further studies evaluating this are needed [31].

Less anticipated was that current smoking status was also associated with a shorter LOS. This may be attributed to the "healthy smoker effect" whereby smokers have less perception or greater tolerance of their symptoms [32, 33]. Further to this, the desire for discharge may be enhanced by additional motivational factors, such as a desire to smoke and therefore to mobilise earlier than ex-smokers. Though the present study did not assess psychosocial factors which have been previously noted to impact LOS [11, 34], this observed effect with smoking status may be related to such factors and should be further evaluated.

\section{Disease and exacerbation severity-, and treatment-related factors}

Disease and exacerbation severity-, and treatment-related factors were more strongly associated with a LOS longer than the median, and of those, the biggest impact was noted with GOLD classification IV. This finding is in keeping with previous reports $[8,35,36]$. A history of previous admissions was also associated with longer LOS. This effect may be due to disease severity but is also likely to be impacted by psychosocial factors. Certainly, an association between LOS, depression and early readmission has been reported [37].

Although severity scores for COPD exacerbation exist, such as BODE index [38], these were not recorded as a variable in the present study. Therefore, other clinical features that occur in more severe episodes have been evaluated and interpreted as surrogate markers of exacerbation severity.

Patients who were acidotic or had sputum purulence were also more likely to have an increased LOS (OR 1.25 and 1.11, respectively) as reported by previous studies in different populations [6-9].

The use of invasive ventilation was associated with increased LOS. In keeping with this is a report by Limsuwat et al. [39], who also found intubation and mechanical ventilation was associated with increased LOS. The use of antibiotics, theophylline and diuretics were also associated with an increased risk of a LOS longer than the median. This may be attributed to a more severe episode, as such factors may be considered surrogate markers of a more severe episode. However, this interpretation is subject to appropriate treatment selection, and does not consider the potential increase in LOS caused by over-treatment that may result in complications such as antibiotic-associated diarrhoea or oxygen-induced hypercapnic respiratory failure, which may impact LOS. 
Variable

\section{Patient-related variables}

Age years

Smoking status

Charlson Comorbidity Index score

Body mass index $\mathrm{kg} \cdot \mathrm{m}^{-2}$

\section{Disease-related variables}

Previous admissions

GOLD stage

\section{Exacerbation severity-related variables}

$\mathrm{pH}$

Purulent sputum

Consolidation

\section{Treatment-related variables}

Invasive mechanical ventilation

In-hospital antibiotics

In-hospital methylxanthines

In-hospital inhaled corticosteroids

In-hospital diuretics

Radiography performed

\section{Resources-related variables}

Respiratory ward

Number of respiratory specialists in unit

\section{Category}

Ex-smoker

Active

Never-smoker

Missing

$<19.5$

$19.5-24.99$

25-29.99

$>29.99$

Missing

No

Yes

Missing

1

2

3

4

Missing

$<7.35$

$\geqslant 7.35$

Missing

No

Yes

Missing

No

Yes

No

Yes

No

Yes

No

Yes

No

Yes

No

Yes

No

Yes

Yes

No

Missing

$<6$

$\geqslant 6$

Missing
OR $(95 \% \mathrm{CI})$

$1.01(1.01-1.02)$

1

0.85 (0.78-0.93)

$0.94(0.80-1.12)$

$1.08(0.92-1.28)$

$1.04(1.01-1.06)$

1.13 (0.97-1.33) 1

$0.89(0.79-1.00)$

$0.88(0.78-1.00)$

0.83 (0.74-0.93)

\section{1}

$1.15(1.07-1.24)$

$1.11(0.96-1.28)$

$$
1
$$

$1.10(0.79-1.55)$

$1.20(0.86-1.67)$

$1.77(1.25-2.49)$

$1.36(0.97-1.90)$

$1.25(1.12-1.40)$ 1

$1.04(0.93-1.16)$

$$
1
$$

$1.11(1.03-1.20)$

$1.05(0.91-1.20)$ 1

$1.27(1.16-1.40)$

$$
1
$$

$2.15(1.25-3.72)$

$1.46(1.30-1.64)$

$$
1
$$

$1.22(1.07-1.40)$

$$
1
$$

$1.10(1.01-1.20)$ 1

$1.36(1.25-1.49)$ 1

$1.42(1.03-1.95)$$$
1
$$

$1.33(1.22-1.45)$

0.38 (0.11-1.32)

$$
1
$$

$1.19(1.00-1.40)$

$0.68(0.38-1.24)$

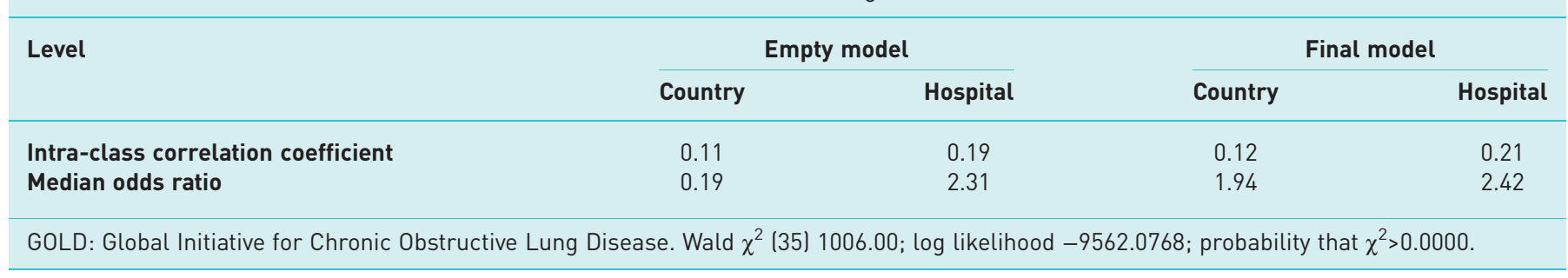



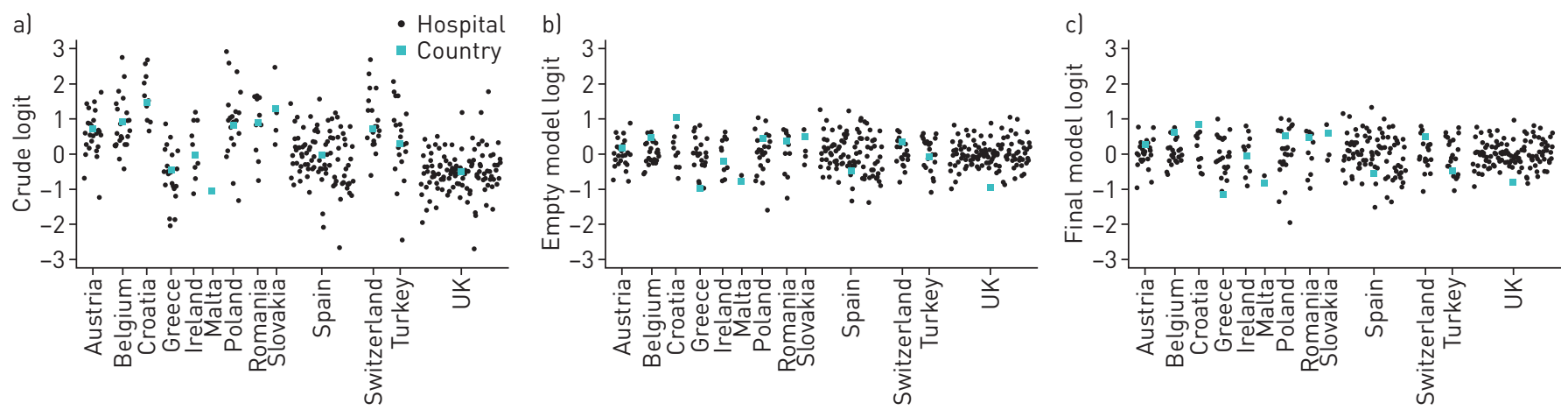

FIGURE 1 The variability of probability of that the length of stay is longer than the median by country. The black dots represent the logit of each hospital $a, b)$ accounting only for cluster effects without covariates and c) accounting for cluster effects and covariates. The green dots represent the logit of each country. Logit is defined as the probability determined by logistic regression: $\operatorname{logit}(p)=\log (p / 1-p)$.

\section{Resource and organisational factors}

Of the resource and organisational factors that were found to be associated with a LOS longer than the median in the initial bivariate analysis, only two were retained in the final model. This was despite taking a more conservative value for significance $(\mathrm{p}>0.1)$. In this analysis, no association was noted between LOS in hospitals where early supported discharge service was available, where there was a respiratory consultant available out of hours or where there were more than six respiratory consultants in the organisation, contrasting with previous reports $[4,14]$. Whilst in the UK, $75.2 \%$ of centres had an early supported discharge service, in the rest of Europe, this figure declined to $15.4 \%$ and is likely to include a number of different service models under that umbrella term.

Data from the UK COPD audit data from 2003 and 2008 [14, 26], very much support the implementation of services such as early supported discharge programmes due to an observed reduction in LOS and these reports suggested that units with more respiratory consultants and better quality organised care had lower mortality and reduced LOS. Furthermore, a previous randomised controlled trial [4] also noted a dramatic reduction in LOS from 6.1 to 3.2 days in a group of patients receiving care from an early supported discharge service versus standard inpatient care. The explanation for our findings is unclear but may relate to the relatively high LOS reported in this audit by the UK centres and the low level of this form of service across Europe as a whole. Other studies have supported use of early supported discharge services in the absence of a reduction in LOS [40-42] due to patient preference or positive impacts on patients' well-being with use of such services.

a)

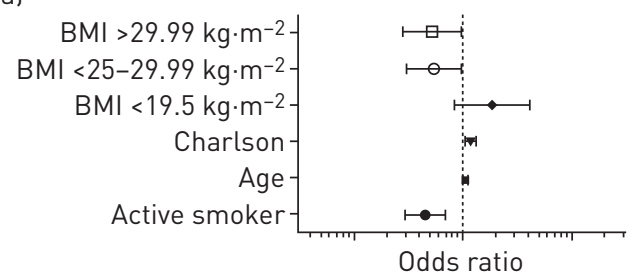

c)

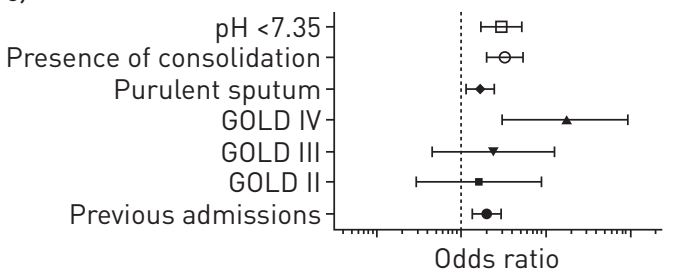

b)

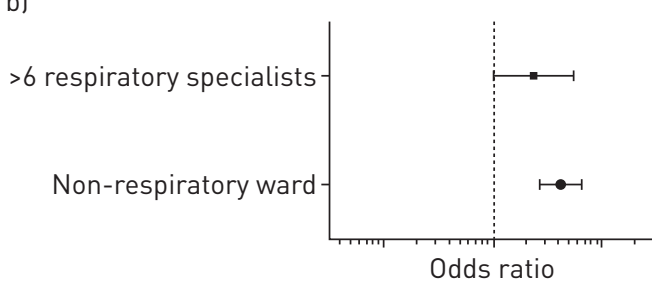

d)

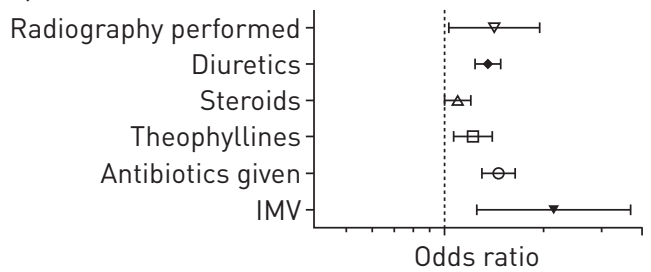

FIGURE 2 Odds ratios for a length of stay longer than the median of the variables retained in the final model. Vertical dashed lines represent OR 1. BMI: body mass index; GOLD: Global Initiative for Chronic Obstructive Lung Disease; IMV: invasive mechanical ventilation. 
This study has found that when resource and organisational factors are considered within a cluster model their individual impact on length of stay is greatly diminished. In real-life situations, it is the complex manner in which these various factors interact that determines outcomes for patients and that is reflected in the cluster analysis. Further studies are needed to evaluate this complex interaction in more detail in order to tease out the important influencing factors and to provide appropriate targets for improving outcomes with relation to length of stay.

\section{Strengths and limitations}

The design of the study and statistical methods underwent a great deal of consideration. Other methods of data analysis, including logit, Poisson, negative and polynomial regression, were rejected as it was felt that the chosen methodology, which enabled accounting for factors that were not measured as outcomes, was superior. Factors such as hospital admission policies, cultural practices, presence of family at home to care for the discharged patients, weather, availability of beds, clinical practice styles, physician thresholds for discharge and socio-demographic factors vary greatly across the countries in Europe included within this audit, and all have an impact on LOS. As none of these was recorded in this study and many are difficult to measure, the multi-level approach was considered ideal, as these factors would be incorporated within the hospital cluster effect.

Use of such a model has obvious limitations in keeping with other mathematical models; for example, it may over-account for factors, making some associations difficult to find. The aim, however, is to highlight the most important and significant associations. Further studies are vital to evaluate the relative importance of the factors found to be associated with LOS in this study and other factors that may be encompassed within the hospital cluster effect that have not been measured.

Participation was on a voluntary basis, so there was no intention of representative sampling. The heterogeneity of the study participants across multiple countries and the interpretation of study variables by different investigators are likely additional limiting factors.

The diagnosis of exacerbation of COPD may be subject to interpretation, and by excluding patients who were admitted for less than $12 \mathrm{~h}$, we perhaps imposed a degree of selection bias into our population, and perhaps it would be also interesting to evaluate those who presented to hospital but were discharged more quickly.

Missing data were a significant problem, as is often the case with retrospective audits. Various methods of dealing this were considered, all of which posed their own problems. We acknowledge that the approach utilised was not flawless, but we considered it the least problematic and, in most cases, the more amenable to clinical interpretation.

Despite these limitations, the strengths of the present study, such as the wide European sampling and the comprehensive statistical analysis of organisational and clinical variables by using a multilevel approach, is highly informative and serves to eliminate biases associated with the clustering effect.

\section{Conclusions}

This study has shown that there is a wide observed variation in LOS across Europe. Some of this is likely to be attributable to the different service models used in those countries. Patient and care process factors of highest impact that were associated with increased LOS in this study were reduced BMI, advanced GOLD classification, presence of respiratory acidosis, consolidation and the use of diuretics. Resource and organisational factors such as size of hospital and the presence of early supported discharge service were not key determinants of LOS after accounting for the clustering effect, whilst admission to a respiratory ward remained an important factor. Identifying patients at risk of a long hospital stay at admission and providing targeted interventions such as early mobilisation offers the potential to reduce LOS for these individuals.

Whilst there are a number of individual factors that affect the LOS of any one patient, it is not just the presence of resources or services but the complex interactions of how a hospital functions to use those resources and services effectively, or not, that is most important in determining the average LOS of a cohort of admitted COPD patients. We suggest that future research should be targeted at identifying those factors that optimise the organisational effectiveness of hospitals irrespective of the health service system within which they operate.

\section{References}

1 Nowak D, Berger K, Lippert B, et al. Epidemiology and health economics of COPD across Europe: a critical analysis. Treatment Respir Med 2005; 4: 381-395.

2 Chapman KR, Mannino DM, Soriano JB, et al. Epidemiology and costs of chronic obstructive pulmonary disease. Eur Respir J 2006; 27: 188-207.

3 Simoens S. Cost-effectiveness of pharmacotherapy for COPD in ambulatory care: a review. J Evalu Clin Pract 2013; 19: 1004-1011. 

pulmonary disease: a randomized controlled trial. Thorax 2000; 55: 902-906.

5 Liu SX, Lee MC, Atakhorrami M, et al. Economic assessment of home-based COPD management programs COPD 2013; 10: 640-649.

6 Agboado G, Peters J, Donkin L. Factors influencing the length of hospital stay among patients resident in Blackpool admitted with COPD: a cross-sectional study. BMJ Open 2012; 2: e000869.

7 Myint PK, Lowe D, Stone RA, et al. U.K. National COPD Resources and Outcomes Project 2008: patients with chronic obstructive pulmonary disease exacerbations who present with radiological pneumonia have worse outcome compared to those with non-pneumonic chronic obstructive pulmonary disease exacerbations. Respiration 2011; 82: 320-327.

8 Nowinski A, Kaminski D, Korzybski D, et al. Wplyw chorob wspolistniejacych na dlugosc leczenia szpitalnegou chorych z zaostrzeniem przewleklej obturacyjnej choroby pluc [The impact of comorbidities on the length of hospital treatment in patients with chronic obstructive pulmonary disease]. Pneumonol Alergol Pol 2011; 79: 388-396.

9 Villalta J, Sequeira E, Cereijo AC, et al. Factores predictivos de un ingreso corto en pacientes con enfermedad pulmonar obstructiva crónica agudizada [Factors predicting a short length of stay for acute exacerbations of chronic obstructive pulmonary disease]. Med Clin 2005; 124: 648-650.

10 Poole PJ, Chase B, Frankel A, et al. Case management may reduce length of hospital stay in patients with recurrent admissions for chronic obstructive pulmonary disease. Respirology 2001; 6: 37-42.

11 Wong AW, Gan WQ, Burns J, et al. Acute exacerbation of chronic obstructive pulmonary disease: influence of social factors in determining length of hospital stay and readmission rates. Can Respir J 2008; 15: 361-364.

12 Lopez-Campos JL, Hartl S, Pozo-Rodriguez F, et al. Variability of hospital resources for acute care of COPD patients: the European COPD Audit. Eur Respir J 2014; 43: 754-762.

13 Quantrill SJ, Lowe D, Hosker HS, et al. Survey of early discharge schemes from the 2003 UK National COPD Audit. Respir Med 2007; 101: 1026-1031.

14 Price LC, Lowe D, Hosker HS, et al. UK National COPD Audit 2003: Impact of hospital resources and organisation of care on patient outcome following admission for acute COPD exacerbation. Thorax 2006; 61: 837-842.

15 Wijayaratne K, Wilson J, Sivakumaran P, et al. Differences in care between general medicine and respiratory specialists in the management of patients hospitalized for acute exacerbations of chronic obstructive pulmonary disease. Ann Thorac Med 2013; 8: 197-203.

16 Shepperd S, Doll H, Broad J, et al. Early discharge hospital at home. Cochrane Database Syst Rev 2009; 0: CD000356.

17 Lu CC, Su HF, Tsay SL, et al. A pilot study of a case management program for patients with chronic obstructive pulmonary disease (COPD). J Nurs Res 2007; 15: 89-98.

18 Lopez-Campos JL, Hartl S, Pozo-Rodriguez F, et al. European COPD Audit: design, organisation of work and methodology. Eur Respir J 2013; 41: 270-276.

19 Charlson ME, Pompei P, Ales KL, et al. A new method of classifying prognostic comorbidity in longitudinal studies: development and validation. J Chronic Dis 1987; 40: 373-383.

20 Pozo-Rodriguez F, Lopez-Campos JL, Alvarez-Martinez CJ, et al. Clinical audit of COPD patients requiring hospital admissions in Spain: AUDIPOC study. PloS One 2012; 7: e42156.

21 Roberts CM. The UK Experience of COPD National Audit. Pneumonol Alergol Pol 2012; 80: 498-500.

22 Snijders TAB, Bosker RJ. Multilevel Analysis: An Introduction to Basic and Advanced Multilevel Modeling. Thousand Oaks, SAGE Publications, 2011.

23 Merlo J. Multilevel analytical approaches in social epidemiology: measures of health variation compared with traditional measures of association. J Epidemiol Commun Health 2003; 57: 550-552.

24 Merlo J, Chaix B, Ohlsson H, et al. A brief conceptual tutorial of multilevel analysis in social epidemiology: using measures of clustering in multilevel logistic regression to investigate contextual phenomena. J Epidemiol Commun Health 2006; 60: 290-297.

25 Hartl S, Lopez-Campos JL, Pozo-Rodriguez F, et al. Risk of death and readmission of hospital-admitted COPD exacerbations: European COPD Audit. Eur Respir J 2015; 47: 113-121.

26 George PM, Stone RA, Buckingham RJ, et al. Changes in NHS organization of care and management of hospital admissions with COPD exacerbations between the national COPD audits of 2003 and 2008. QJM 2011; 104: 859-866.

27 Busby J, Purdy S, Hollingworth W. A systematic review of the magnitude and cause of geographic variation in unplanned hospital admission rates and length of stay for ambulatory care sensitive conditions. BMC Health Serv Res 2015; 15: 324 .

28 Roberts CM, Stone RA, Lowe D, et al. Co-morbidities and 90-day outcomes in hospitalized COPD exacerbations. COPD 2011; 8: 354-361.

29 Collins PF, Elia M, Stratton RJ. Nutritional support and functional capacity in chronic obstructive pulmonary disease: a systematic review and meta-analysis. Respirology 2013; 18: 616-629.

30 Pooler A, Beech R. Examining the relationship between anxiety and depression and exacerbations of COPD which result in hospital admission: a systematic review. Int J Chron Obstruct Pulmon Dis 2014; 9: 315-330.

31 Snider JT, Jena AB, Linthicum MT, et al. Effect of hospital use of oral nutritional supplementation on length of stay, hospital cost, and 30-day readmissions among Medicare patients with COPD. Chest 2015; 147: $1477-1484$.

32 Cerveri I, Cazzoletti L, Corsico AG, et al. The impact of cigarette smoking on asthma: a population-based international cohort study. Int Arch Allergy Immunol 2012; 158: 175-183.

33 Becklake MR, Lalloo U. The 'healthy smoker': a phenomenon of health selection? Respiration 1990; 57: 137-144.

$34 \mathrm{Ng}$ TP, Niti M, Tan WC, et al. Depressive symptoms and chronic obstructive pulmonary disease: effect on mortality, hospital readmission, symptom burden, functional status, and quality of life. Arch Intern Med 2007; 167: 60-67.

35 Zampieri FG, Ladeira JP, Park M, et al. Admission factors associated with prolonged ( $>14$ days) intensive care unit stay. J Crit Care 2014; 29: 60-65.

36 Incalzi RA, Pedone C, Onder G, et al. Predicting length of stay of older patients with exacerbated chronic obstructive pulmonary disease. Aging (Milano) 2001; 13: 49-57.

37 Sharif R, Parekh TM, Pierson KS, et al. Predictors of early readmission among patients 40 to 64 years of age hospitalized for chronic obstructive pulmonary disease. Ann Am Thorac Soc 2014; 11: 685-694.

38 Moberg M, Vestbo J, Martinez G, et al. Validation of the i-BODE index as a predictor of hospitalization and mortality in patients with COPD participating in pulmonary rehabilitation. COPD 2014; 11: 381-387. 
39 Limsuwat C, Mankongpaisarnrung C, Dumrongmongcolgul N, et al. Factors influencing the length of hospital stay in patients with acute exacerbations of chronic obstructive pulmonary disease admitted to intensive care units. Qual Manag Health Care 2014; 23: 86-93.

40 Utens CM, Goossens LM, Smeenk FW, et al. Early assisted discharge with generic community nursing for chronic obstructive pulmonary disease exacerbations: results of a randomised controlled trial. BMJ Open 2012; 2: e001684.

41 Wang Y, Haugen T, Steihaug S, et al. Patients with acute exacerbation of chronic obstructive pulmonary disease feel safe when treated at home: a qualitative study. BMC Pulm Med 2012; 12: 45.

42 Utens CM, Goossens LM, van Schayck OC, et al. Patient preference and satisfaction in hospital-at-home and usual hospital care for COPD exacerbations: results of a randomised controlled trial. Int J Nurs Stud 2013; 50: 1537-1549. 